\title{
Biomembranes from Slaughterhouse Blood Erythrocytes as Prolonged Release Systems for Dexamethasone Sodium Phosphate
}

\section{Ivana T. Drvenica}

Dept. of Chemical Engineering, Faculty of Technology and Metallurgy, University of Belgrade, Belgrade, Serbia

Katarina M. Bukara

Dept. of Chemical Engineering, Faculty of Technology and Metallurgy, University of Belgrade, Belgrade, Serbia

\section{Vesna Lj. Ilić}

Inst. for Medical Research, University of Belgrade, Belgrade, Serbia

\section{Danijela M. Mišić}

Inst. for Biological Research "Siniša Stanković,”, University of Belgrade, Belgrade, Serbia

Borislav Z. Vasić

Inst. of Physics, University of Belgrade, Belgrade, Serbia

\section{Radoš B. Gajić}

Inst. of Physics, University of Belgrade, Belgrade, Serbia

\section{Verica B. Đordević}

Dept. of Chemical Engineering, Faculty of Technology and Metallurgy, University of Belgrade, Belgrade, Serbia

\section{Đorde N. Veljović}

Dept. of Chemical Engineering, Faculty of Technology and Metallurgy, University of Belgrade, Belgrade, Serbia

\author{
Aleksandar Belić \\ Inst. of Physics, University of Belgrade, Belgrade, Serbia
}

\section{Branko M. Bugarski}

Dept. of Chemical Engineering, Faculty of Technology and Metallurgy, University of Belgrade, Belgrade, Serbia

The present study investigated preparation of bovine and porcine erythrocyte membranes from slaughterhouse blood as bio-derived materials for delivery of dexamethasone-sodium phosphate (DexP). The obtained biomembranes, i.e., ghosts were characterized in vitro in terms of morphological properties, loading parameters, and release behavior. For the last two, an UHPLC/-HESI-MS/MS based analytical procedure for absolute drug identification and quantification was developed. The results revealed that loading of DexP into both type of ghosts was directly proportional to the increase of drug concentration in the incubation medium, while incubation at $37^{\circ} \mathrm{C}$ had statistically significant effect on loaded amount of DexP $(P<0.05)$. The encapsulation efficiency was about fivefold higher in porcine compared to bovine ghosts. Insight into ghosts' surface morphology by field emission-scanning electron microscopy and atomic force microscopy confirmed that besides inevitable effects of osmosis, DexP inclusion itself had no observable additional effect on the morphology of the ghosts carriers. DexP release profiles were dependent on erythrocyte ghost type and amount of residual hemoglobin. However, sustained DexP release was achieved and shown over 3 days from porcine ghosts and 5 days from bovine erythrocyte ghosts. (C) 2016 American Institute of Chemical Engineers Biotechnol. Prog., 32:1046-1055, 2016

Keywords: biomembranes, erythrocyte ghosts, slaughterhouse blood, dexamethasone sodium phosphate, drug delivery systems

Additional Supporting Information may be found in the online version of this article.

Correspondence concerning this article should be addressed to I. T. Drvenica at ikostic@tmf.bg.ac.rs.

\section{Introduction}

Dexamethasone is a potent, synthetic member of glucocorticoid class of steroids with anti-inflammatory and immunosuppressive features. It is intensively used in therapy of 
inflammatory conditions but with a frequent dosage, due to the short elimination half time of approximately $3 \mathrm{~h}$. This causes various adverse effects and low compliance, especially in chronic therapy. ${ }^{1}$ Beside therapeutic application, dexamethasone is widely employed as a component of cell culture media; however, the direct use of dexamethasone in the culture medium has been also limited, mainly due to toxic side effects. ${ }^{2,3}$ It has been shown that all mentioned side effects of dexamethasone application could be avoided if the drug is administered in a controlled or extended manner ${ }^{3-7}$; therefore, the continuous development of delivery systems which are able to achieve sustainable release of dexamethasone is needed.

Synthetic drug carriers, such as polymer and lipid particles, often struggle to meet clinical expectations. ${ }^{8}$ In contrast, biological particulates that range from bacterial to mammalian cells are especially suitable for in vivo applications and they are also able to provide controlled drug delivery. ${ }^{8}$ The systems for controlled delivery of dexamethasone made from human erythrocytes have been explored for many years. In favor of the great importance of this kind of biological carriers is the fact about several successful clinical trials done so far. ${ }^{9-14}$ It should be emphasized that all examined erythrocyte-based systems deliver dexamethasone in lower doses than conventional formulations, and thus provide clinical benefit proper for corticosteroid treatment without the toxic side effects. ${ }^{15}$

From a pharmacokinetic and pharmacodynamics perspective, the use of autologous human erythrocytes as carrier systems for dexamethasone targeted delivery seems to give satisfying results in personal medicine. ${ }^{15}$ However, in order to produce universal carrier for delivery of dexamethasone, the use of heterologous (allogeneic) human red blood cells are less acceptable due to the limited amount of red blood cells available for production of the formulations. Another limiting fact is the absence of a biologically safe, large scale procedure for preparing these kinds of carriers. The engineering of other biological materials through the utilization and mimicry of bacteria and eukaryotic cells is the possible solution. ${ }^{16}$ It goes in line with the studies of protocols for surface modifications and covering antigenic determinates on cell membranes in order to attenuate the hosts' immune response to incompatible erythrocytes. ${ }^{17,18}$

Particularly, erythrocytes from slaughterhouse blood should be taken into consideration as a high amount is produced on a daily basis. Beside some reports on hemoglobin isolation and its enzymatic modifications, ${ }^{19-21}$ other applications of cellular fractions of bovine and porcine slaughterhouse blood, such as erythrocyte ghosts, are scarce. ${ }^{22,23}$ The erythrocyte membranes have been successfully isolated from bovine and porcine slaughterhouse blood by a pilot-scale optimized gradual hypotonic hemolysis in our recent study, ${ }^{24}$ and the ghosts were characterized in terms of their biochemical and morphological properties. Furthermore, in the same study it was shown that the gradual hemolysis can be used for loading of empty membranes (ghosts) with drugs-dexamethasone sodium phosphate (DexP) was used a model drug. With the aim of developing DexP vehicle, in this study we further examined the conditions of the gradual hemolysis (initial drug concentration, incubation temperature, and the addition of crosslinking agent).

Within the development of novel drug delivery systems, there are two main analytical aspects to take into account: quantification of drug trapped into the carriers and quantifi- cation of drug released from the carriers. ${ }^{25}$ It means that procedure for drug carrier breakage must be established to ensure the total extraction of the drug. Furthermore, extracted drug must be quantified in a specific, accurate, and reproducible way. ${ }^{26}$ Regarding the quantification of the released drug, the developed analytical procedure must enable detection of low drug amounts in the dissolution medium and selectively quantify them in the presence of other delivery system constituents also dissolved in the medium. ${ }^{25}$ Since the chemical structure of erythrocyte ghosts is rather complex (composed of lipids, proteins, and carbohydrates) and encapsulated DexP can be dephosphorylated to dexamethasone (Dex) by erythrocyte-resident enzymes, ${ }^{15}$ an essential requirement for this study was development of an analytical procedure for absolute drug identification and quantification. Herein, we have developed an UHPLC/HESI-MS/MS based analytical procedure.

Besides the precise quantification of encapsulated drug, we investigate the DexP-loaded biomembranes going in detail into their morphological and release properties.

\section{Materials and Methods}

\section{Chemicals}

The salts used in the preparation of buffers, and dexamethasone (Dex) standard were of analytical grade and purchased from Sigma-Aldrich (St. Louis, MO). Dexamethasone sodium phosphate (DexP) solution for injection $(4 \mathrm{mg} / \mathrm{mL})$ was supplied from Galenika a.d., Serbia. Isotonic phosphate buffered saline solution (PBS, $\mathrm{pH}$ 7.4) containing $137 \mathrm{mM}$ sodium chloride and $10 \mathrm{mM}$ phosphate buffer was prepared from salts and distilled water. Hypertonic phosphate PBS $(2 \times \mathrm{PBS})$ was prepared as described for PBS except for the addition of two times the $\mathrm{NaCl}$ concentration. The osmolality of the phosphate buffers was measured using Osmomat 030 Osmometer (Gonotec, Germany). The glutaraldehyde (GA) solution (50\% in water) was from ChemikaFluka (Buchs, Switzerland). All solvents were from Merck, Darmstadt, Germany with HPLC purity grades, whenever needed.

\section{Blood samples and preparation of packed erythrocytes}

The present study was performed utilizing bovine and porcine blood, collected during slaughtering, in slaughterhouse "PKB Imes" in Belgrade, Serbia. Blood of Holstein-Friesian calves and Swedish Landrace swine was taken from jugular vein and collected in a sterile glass bottle containing $3.8 \%$ $\mathrm{Na}$-citrate as an anticoagulant agent. Preparations of packed erythrocytes, as well as determination of hemoglobin concentration, hematocrit, erythrocyte number, and remained hemoglobin content in the ghosts were performed as described in Kostić et al. ${ }^{24}$

\section{Encapsulation of DexP in erythrocyte ghosts}

A gradual hypotonic hemolysis method ${ }^{24}$ using a very simple set up was employed for DexP loading into bovine and porcine erythrocyte ghosts. For this purpose, $50 \mathrm{~mL}$ washed packed erythrocytes were suspended in isotonic PBS to an Hct of $60 \%$, and poured in a $2 \mathrm{~L}$ glass beaker. Afterward, the hypotonic PBS of $70 \mathrm{mOsm} / \mathrm{kg}$ containing $0.04 \mathrm{mg} / \mathrm{mL}$ DexP was introduced gradually by infusion pump with a flow rate of $150 \mathrm{~mL} / \mathrm{h}$ during $27 \mathrm{~min}$. The 
ghosts were separated by centrifugation and washed with the hypotonic PBS four times. The relative quantity of hemoglobin which remained within the erythrocyte membranes was expressed as described in Kostić et al. ${ }^{24}$ Then, $1 \mathrm{~mL}$ of ghosts was annealed in $8 \mathrm{~mL}$ of isotonic PBS $(30 \mathrm{~min}$, at room temperature $\left(25^{\circ} \mathrm{C}\right.$ ) or $37^{\circ} \mathrm{C}$ ), containing DexP (at concentrations of $0.04,0.08$, or $0.12 \mathrm{mg} / \mathrm{mL}$ ). Afterward, the same volume of ghosts were transferred to $8 \mathrm{~mL}$ of hypertonic buffer containing the same DexP concentration as well as GA in concentration of $0.01 \%(\mathrm{v} / \mathrm{v})$ and incubated for 90 min at 25 and $37^{\circ} \mathrm{C}$. Finally, the ghost carriers were washed three times using PBS by centrifugation to wash out the unentrapped DexP. In some experiments sham-encapsulated ghosts (i.e., the erythrocytes under complete loading procedure with no drug added) were needed. These were prepared as described except that the DexP aqueous solution was replaced by drug-free buffer solution.

\section{Samples preparation for UHPLC/DAD/-HESI-MS/MS analysis}

The loaded ghosts $(0.1 \mathrm{~mL})$ were lysed by diluting with $2 \mathrm{~mL}$ of distilled water followed by the addition of $2 \mathrm{~mL}$ of methanol for deproteinization as given in Hamidi et al. ${ }^{27}$ The resulting mixture was kept for $30 \mathrm{~min}$, at $25^{\circ} \mathrm{C}$, and then centrifuged at $14,800 \mathrm{rpm}$ for $15 \mathrm{~min}$. The supernatant was filtered through $0.22 \mu \mathrm{m}$ syringe filters and $10 \mu \mathrm{L}$ of supernatant was injected to chromatograph.

\section{Drug assay}

An UHPLC/DAD/-HESI-MS/MS method was developed to identify and quantify DexP concentrations in samples of loaded ghosts. Separation, determination, and quantification of DexP in samples was performed using Dionex Ultimate 3000 UHPLC system (ThermoFisher Scientific, Bremen, Germany) equipped with a diode array detector (DAD), and connected to TSQ Quantum Access Max triple-quadrupole mass spectrometer (ThermoFisher Scientific, Basel, Switzerland). Elution was performed at $30^{\circ} \mathrm{C}$ on Hypersil gold $\mathrm{C} 18$ column $(50 \times 2.1 \mathrm{~mm})$ with $1.9 \mu \mathrm{m}$ particle size (ThermoFisher Scientific). The mobile phase consisted of (i) water$+0.1 \%$ formic acid and (ii) acetonitrile (MS grade, Fisher Scientific UK, Leics, UK), which were applied in the gradient elution described by Kostić et al. ${ }^{24}$ The flow rate was set to $0.4 \mathrm{~mL} / \mathrm{min}$, and the detection wavelength to $250 \mathrm{~nm}$. The column was thermostated at $30^{\circ} \mathrm{C}$. The injection volume was $10 \mu \mathrm{L}$. All analyses were performed in triplicate.

A TSQ Quantum Access Max triple-quadrupole mass spectrometer equipped with an heated electrospray ionization (HESI) source was used with vaporizer temperature kept at $450^{\circ} \mathrm{C}$, and ion source settings as follows: spray voltage 4,000 V, sheet gas $\left(\mathrm{N}_{2}\right)$ pressure $50 \mathrm{AU}$, ion sweep gas pressure $0 \mathrm{AU}$, auxiliary gas $\left(\mathrm{N}_{2}\right)$ pressure $20 \mathrm{AU}$, capillary temperature at $320^{\circ} \mathrm{C}$, and skimmer offset $0 \mathrm{~V}$. Mass spectrometry data were acquired in both positive and negative mode, in $\mathrm{m} / \mathrm{z}$ range from 100 to 1,000 . Multiple mass spectrometric scanning modes, including full scanning (FS) and product ion scanning (PIS) were conducted for the qualitative analysis. Collision-induced fragmentation experiments were performed using argon as the collision gas, and collision energy was set to $40 \mathrm{eV}$. Selected reaction monitoring (SRM) experiment for quantitative analysis was performed by using two $\mathrm{MS}^{2}$ fragments for DexP, which were previ- ously defined as dominant in PIS experiments. Quantification of DexP was based on the calibration curve of pure compound. Total amount of DexP in each sample was evaluated by the calculation of peak areas and is expressed as $\mathrm{ng} / \mathrm{mL}$. Chromeleon Xpress software (Thermo Fisher Scientific, Bremen, Germany) was used for the data acquisition and method/run control. The identification of DexP in samples was supported by DAD analysis, using Dex and DexP as standards.

\section{Loading parameters}

To evaluate the drug loading in the encapsulated erythrocyte ghosts, two indices were defined as loading parameters, as described in Hamidi et al. ${ }^{27}$ These were loaded amount (the amount of DexP entrapped in $0.1 \mathrm{~mL}$ of the final packed erythrocytes) and encapsulation efficiency (EE, the percentage ratio of the loaded amount of DexP, to the amount expected if the drug entered the ghosts freely in the encapsulation stage and then was completely retained within the ghosts).

\section{Drug release}

In each $1.5 \mathrm{~mL}$ polypropylene microtubes, $100 \mu \mathrm{L}$ of DexP-loaded ghosts were incubated at $37^{\circ} \mathrm{C}$ with $900 \mu \mathrm{L}$ of isotonic PBS and shaken at $240 \mathrm{rpm}$. At the beginning of the test and after defined time intervals the samples were harvested and after centrifuging at 14,800 rpm for $3 \mathrm{~min}, 100$ $\mu \mathrm{L}$ of the supernatant was used for DexP assay. These experiments were carried out in triplicate and the percent of DexP were determined in reference to a completely lysed sample (100\% release).

\section{Field emission-scanning electron microscopy (FE-SEM)}

Morphological changes of erythrocyte ghosts after loading procedure were analyzed by field emission scanning electron microscope (FE-SEM). The samples for SEM were prepared as described in Kostić et al. ${ }^{24}$

\section{AFM analysis}

The samples of sham- and DexP-encapsulated bovine and porcine erythrocyte ghosts were prepared for the atomic force microscopy (AFM) measurements by introducing the next protocol: $500 \mu \mathrm{L}$ of erythrocytes and ghosts were allowed to settle onto poly-L-lysine-coated cover glasses at $4^{\circ} \mathrm{C}$ overnight. Samples were fixed in $2 \%$ GA in PBS and $2 \%$ osmium tetroxide for $2 \mathrm{~h}$ each, washed, and dehydrated through a graded series of ethanol solutions (10, 30, 50, 70, 95 , and $100 \%$ ) for $10 \mathrm{~min}$ each. The final dehydration step consisted of three times washing in acetone. Topographic AFM measurements were performed in the semicontact mode under ambient conditions using the NTEGRA Prima system from NT-MDT (NT-MDT Co. Moscow, Russia). Simultaneously with the topographic measurements, the phase lags of the AFM cantilever oscillations were recorded thus forming AFM phase images. NSG01 probes from NTMDT with a typical tip curvature radius of about $6 \mathrm{~nm}$ and a typical force constant of $5 \mathrm{~N} / \mathrm{m}$ were used. Topographic images were processed using the instrument-equipped software by the plane subtraction or by the fitting with the firstorder lines, while the raw data was used for the phase images. For the calculation of the root-mean-square (RMS) 
of the surface roughness, only cell surfaces were selected from AFM images and then RMS was directly generated by the instrument software, using a total of 15 cell measurements for each ghost preparations.

\section{Data analysis and statistics}

The results are presented as mean \pm standard deviation (SD). The statistical significance of the differences was analyzed by two tailed paired $t$-test. The level of significance $\mathrm{p}$ was taken as 0.05 in all cases. All analyses were done using Microsoft Office Excel 2007 software (Microsoft Corporation, WA).

\section{Results and Discussion}

Herein, we have developed an UHPLC/-HESI-MS/MS based analytical procedure for a precise quantification of encapsulated DexP and the release kinetics. Prior to quantitative analysis, the confirmation of the presence of DexP after encapsulation in bovine and porcine erythrocyte ghosts, Fourier transform infrared spectroscopy (FTIR) analysis was conducted and given in the Supporting Information.

\section{Drug assay}

Initially, UHPLC/-HESI-MS total ion chromatogram (TIC) of pure DexP (Figure 1A, part 1) showed peak at Rt $5.31 \mathrm{~min}$ with base peak ion at $\mathrm{m} / \mathrm{z} 471$ (Figure 1A, part 2), corresponding to the quasi-molecular ion $[\mathrm{M}+\mathrm{H}-2 \mathrm{Na}]^{-}$. MS/ MS analysis of the precursor ion at $\mathrm{m} / z 471$ gave following major $\mathrm{MS}^{2}$ fragments: $\mathrm{m} / \mathrm{z}$ 471, $\mathrm{m} / \mathrm{z} 453, \mathrm{~m} / \mathrm{z} 441, \mathrm{~m} / \mathrm{z}$ 139, and $\mathrm{m} / \mathrm{z} 97$ (Figure 1A, part 3). In some previous studies, DexP was also clearly characterized by its specific ions $[\mathrm{M}+\mathrm{H}-2 \mathrm{Na}]^{-}$at $\mathrm{m} / z$ 471, $\left[\mathrm{M}+\mathrm{H}-2 \mathrm{Na}-\mathrm{H}_{2} \mathrm{O}\right]^{-}$at $\mathrm{m} / z$ 453, and $\left[\mathrm{M}+\mathrm{H}-2 \mathrm{Na}-\mathrm{CH}_{2} \mathrm{O}\right]^{-}$at $\mathrm{m} / z 441$. $^{28}$

UHPLC/-HESI-MS TIC of the pure Dex (Figure 1A, part 4) showed peak at $R t=5.96$ min with base peak ion at $\mathrm{m} / \mathrm{z}$ 436 (Figure 1A, part 5), corresponding to the formic acid adduct $[\mathrm{M}-\mathrm{H}+\mathrm{HCOO}]^{-}$, which is a result of using formic acid as a mobile phase. MS/MS analysis of the precursor ion at $\mathrm{m} / \mathrm{z} 436$ gave major $\mathrm{MS}^{2}$ fragments at $\mathrm{m} / \mathrm{z} 326, \mathrm{~m} / \mathrm{z} 311$, $\mathrm{m} / \mathrm{z} 307, \mathrm{~m} / \mathrm{z} 293$, and $\mathrm{m} / \mathrm{z} 186$ (Figure 1A, part 6). Therefore, Dex and DexP were clearly chromatographically separated and could be also distinguished according to their MS and MS/MS spectra.

Simple, fast, and reliable UHPLC/DAD/-HESI-MS/MS method for the routine identification and quantification of DexP in samples was developed and validated. DexP in samples was assessed in SRM experiment, which is the most sensitive mode of the triple quadrupole instrument, by the presence of two diagnostic fragment ions, $\mathrm{m} / \mathrm{z} 471$ and $\mathrm{m} / \mathrm{z}$ 97 (Figure 1B). Linear regression analyses of DexP calibration curve revealed an excellent linearity within the range of 2.5-1,000 ng/mL with a correlation coefficient of $r^{2}=0.9999, P<0.001$, limits of detection (LOD) and quantification (LOQ) of the method were 0.1 and $2.5 \mathrm{ng} / \mathrm{mL}$, respectively. Within- and between-run variations of the method throughout the linear range were all less than $10 \%$ with the corresponding accuracies of more than $90 \%$ in all cases. The release of DexP from bovine and porcine ghost samples was also simultaneously monitored by DAD analyses. The presence of Dex was not recorded in either bovine or porcine ghosts using developed UHPLC/DAD/-HESI-
MS/MS method, indicating the complete recovery of the drug loaded in ghosts (DexP). Furthermore, this result demonstrates the absence of resident erythrocyte enzymes in the obtained ghosts, which could convert DexP into Dex. ${ }^{15}$

Therefore, the analytical procedure developed here allowed us to unambiguously identify and quantify DexP extracted from ghosts, to optimize the loading parameters (initial drug concentration, incubation temperature, GA addition, etc.), as well to track DexP release kinetics.

\section{Encapsulation of DexP in bovine and porcine erythrocyte ghosts}

The study investigates effect of temperature, GA addition, as well as drug concentration on the process of DexP loading into bovine and porcine erythrocyte ghosts as a trial to obtain DexP prolonged release systems. The effect of different DexP concentrations and temperature of incubation on the drug loaded amount in bovine and porcine erythrocyte ghosts is shown in Figure 2.

Regardless the added drug concentration and type of erythrocyte ghosts, incubation at $37^{\circ} \mathrm{C}$ had statistically significant stimulating effect on loaded amount of DexP in erythrocyte ghosts $(P<0.05)$. More pronounced effect of incubation at $37^{\circ} \mathrm{C}$ on loading of DexP in erythrocyte ghosts was shown in the case of bovine erythrocyte ghosts, where the amount of loaded DexP was $\sim 18$ times higher compared to that obtained after incubation at room temperature (at the same initial drug feeding of $0.08 \mathrm{mg} / \mathrm{mL}$ ) (Figure 2A).

Obviously, under the same experimental conditions approximately five times higher values of loaded DexP were obtained in the case of porcine compared to bovine erythrocyte ghosts. This result could be explained by difference in membrane lipids and proteins composition between the two types of cells. ${ }^{24,29-31}$ Namely, in mammals, phosphatidylcholine (PC) is generally the most abundant erythrocyte membrane phospholipid. However, there is a striking deviation in bovine erythrocyte membranes which are particularly deficient in PC, and instead of PC they contain high levels of sphingomyelin (SM). ${ }^{32}$ It can be hypothesized that this particular compound reduced membrane permeability of bovine erythrocyte ghosts in contrast to porcine erythrocyte ghosts. This effect of SM was shown on liposomes (biomembrane analogues) via slower drug release from liposomes containing SM compared to liposomes without SM. ${ }^{33}$

Also, the results demonstrated that the loading of DexP into both type of erythrocyte ghosts was directly proportional to the increase of drug concentration in the incubation medium, in the $0.04-0.12 \mathrm{mg} / \mathrm{mL}$ concentration range (Figures 2A,B). This finding is in agreement with the results reported by other research groups, ${ }^{34-37}$ who employed hypoosmotic procedures to encapsulate various drugs in human erythrocyte ghosts.

Figure 2C summarizes the effect of different DexP concentrations at $37^{\circ} \mathrm{C}$ on drug encapsulation efficacy in bovine and porcine erythrocyte ghosts. Under the same conditions of DexP loading procedure, encapsulation of DexP was approximately five times more efficient in porcine ghost compared to bovine ghosts. The initial DexP concentration had no significant influence on the EE, and its recorded value was $13-14 \%$ and $2-3 \%$ for the porcine and bovine erythrocyte ghosts, respectively. 

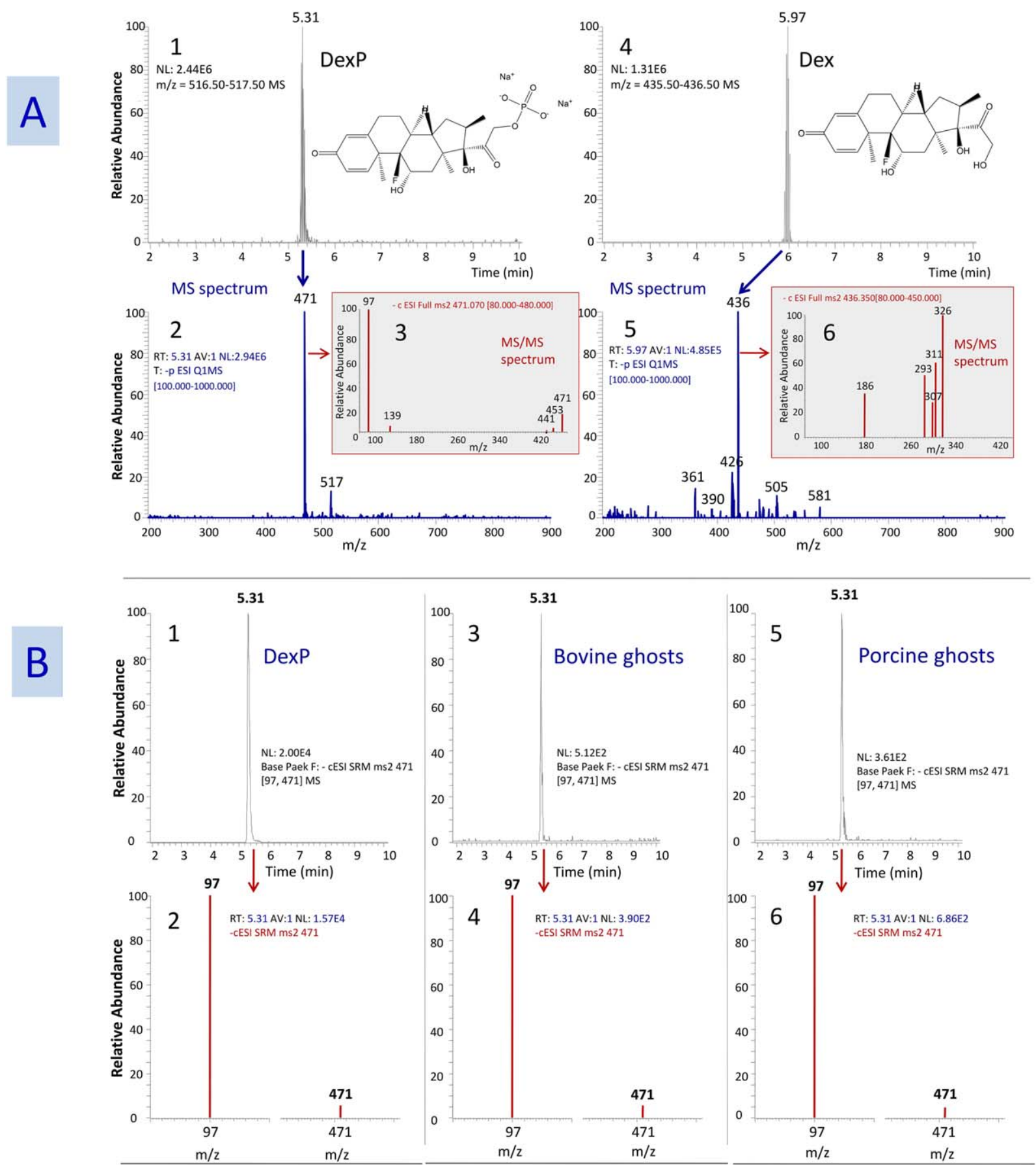

Figure 1. (A) TIC of pure DexP (1) and Dex (4) showed peaks at Rt 5.31 min and Rt 5.97 min, respectively. Mass spectrum of DexP (2) showed base peak ion at $\mathrm{m} / z$ 471, corresponding to the quasi-molecular ion $[\mathrm{M}+\mathrm{H}-2 \mathrm{Na}]^{-}$, while $\mathrm{MS} / \mathrm{MS}$ spectrum (3) showed $\mathrm{MS}^{2}$ fragments at $\mathrm{m} / \mathrm{z} 471, \mathrm{~m} / \mathrm{z} 453, \mathrm{~m} / \mathrm{z} 41, \mathrm{~m} / \mathrm{z}$ 139, and $\mathrm{m} / \mathrm{z}$ 97. Dex MS spectrum (5) showed base peak ion at $\mathrm{m} / \mathrm{z}$ 436, corresponding to the formic acid adduct $[\mathrm{M}-\mathrm{H}+\mathrm{HCOO}]^{-}$, and its $\mathrm{MS} / \mathrm{MS}$ analysis (6) gave major $\mathrm{MS}^{2}$ fragments at $\mathrm{m} / \mathrm{z} 326, \mathrm{~m} / \mathrm{z} 311, \mathrm{~m} / \mathrm{z} 307, \mathrm{~m} / \mathrm{z} 293$, and $\mathrm{m} / \mathrm{z}$ 186. (B) Single reaction monitoring (SRM) chromatograms (upper row) and corresponding mass spectrums (lower row) of DexP in pure $10 \mu \mathrm{g} / \mathrm{mL}$ DexP sample (1 and 2), DexP-loaded bovine ghosts $(3$ and 4), and DexP-loaded porcine ghosts (5 and 6).

The results obtained for DexP loaded porcine erythrocyte ghosts were comparable (in the same range) to the results published for loading different active compounds (drugs, enzymes, proteins, polysaccharides, and DNA) in human erythrocytes ghosts by hypoosmotic procedures. ${ }^{27,36,38-40}$ Lower EE attained in the case of bovine erythrocyte ghosts were in the same range as reported by Kim and co-workers ${ }^{41}$ for AS-ODN encapsulated in human erythrocyte ghosts by loading procedure where hypotonic and isotonic phosphate buffers were employed. Contrary to this, Sternberg et al. $^{42}$ reported approximately $97 \% \mathrm{EE}$ of fluorescein isothiocyanate labelled bovine serum albumin (FITC-BSA) in hemoglobin free human erythrocyte ghosts, where also phosphate buffers were applied in the loading procedure. A possible solution for the problem of low EE could be optimized hypotonic solution/ resealing buffer combination. Thus, Kim and co-workers ${ }^{41}$ highlighted the potential importance of hypotonic buffer composition in the encapsulation process. Furthermore, considering 

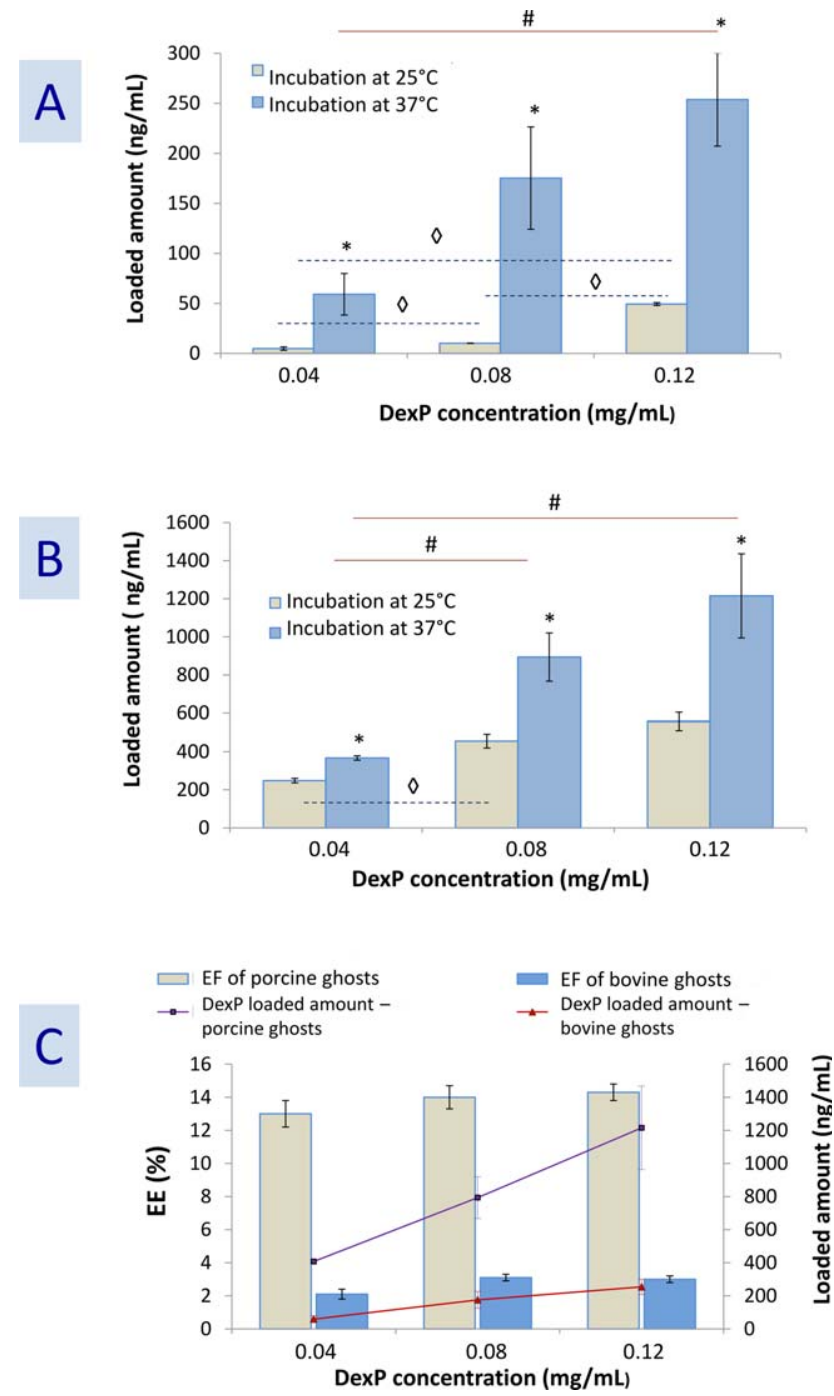

Figure 2. Effects of temperature and drug concentration on the loading amount of DexP in (A) bovine and $(B)$ porcine erythrocyte ghosts. (C) Effect of DexP concentration on encapsulation efficiency at $37^{\circ} \mathrm{C}$ in bovine and porcine erythrocyte ghosts. Values are mean $\pm S D(n=3) ; *$ shows significant difference in DexP loading amount obtained after incubation at 37 and $25^{\circ} \mathrm{C}$; $\square$ and \# shows significant difference in DexP loading amount detected after incubation at 25 or at $37^{\circ} \mathrm{C}$, respectively. Significance determined by $t$-test; $P<0.05$ taken as significant. (C) Effect of DexP concentration on $\mathrm{EE}$ at $37^{\circ} \mathrm{C}$ in bovine and porcine erythrocyte ghosts. Values are mean \pm SD $(n=3)$.

other drug delivery system, our data were consistent with the loading efficiency of DexP reported to be $4-13 \%$ when encapsulated in poly lactic-co-glycolic acid (PLGA) and poly lacticco-glycolic acid/polylactic acid (PLGA/PLA) microspheres. ${ }^{6,43}$

Although no significant difference in EE by adding the different drug concentrations has been shown (Figure 2C), the displayed statistical significance of concentration of $0.12 \mathrm{mg} / \mathrm{mL}$ of DexP and incubation at $37^{\circ} \mathrm{C}$ on drug loading amount (Figures 2A,B) determined these parameters as most favorable. The addition of GA aqueous solution $(0.01 \%$ [v/ v]) in hypertonic stage of loading procedure showed slight effect on improvement of DexP loading parameters (26.5 vs. $34.5 \mathrm{ng} / \mathrm{mL}$ and $144.3 \mathrm{vs} .193 .8 \mathrm{ng} / \mathrm{mL}$ for bovine and porcine erythrocyte ghosts without and with GA, respectively;
$P>0.05)$. This was in contrast to the report of Foroozesh et al. ${ }^{37}$ in which GA $(5 \mu \mathrm{L} / 100 \mathrm{~mL})$ played a crucial role in improvement of drug (tramadol) loading parameters.

\section{Scanning electron microscopy}

In Figure 4 the scanning electron micrographs of shamencapsulated and DexP-loaded bovine and porcine ghosts are shown. SEM images of the both types of loaded ghosts (Figures $3 \mathrm{~B}, \mathrm{D})$ reveal that obtained erythrocyte ghost carriers still resemble the shape of controlled, sham-encapsulated (Figures 3A,C) as well as that of the unloaded and untreated (by loading procedure) ghosts presented in the study of Kostić et al. ${ }^{24}$ The same results were obtained by flow cytometry analysis (see Supporting Information Figure S2). This indicates that DexP loading procedure induce only minor differences in shape and topology of both type of the ghosts. However, the presence of these minor invaginations of DexP encapsulated ghosts compared to sham-encapsulated ghosts were more pronounced in the case of porcine ghosts. This result was in accordance with already reported more susceptible nature of porcine erythrocyte ghosts upon exposure to hypoosmotic conditions. ${ }^{24}$

\section{AFM analysis}

To get more detailed insight into the morphology, i.e., surface topology of DexP loaded bovine and porcine erythrocyte ghosts, we performed AFM analysis. Compared to FE-SEM analysis which provided the qualitative morphological characterization of the ghosts, AFM analysis allows quantification of morphological changes throughout calculation of RMS values. ${ }^{44}$ Results of the AFM morphological analysis are given in Figures 4A,B for DexP loaded bovine and porcine erythrocyte ghosts, respectively, where three-dimensional topographic images (parts A2 and B2) and height cross-section (parts A4 and B4) along the selected lines are given.

During moving of AFM probe along sample surface, both amplitude and phase of the AFM cantilever oscillations are influenced by topographic features. In the vicinity of sharp steps in the topography, AFM phase changes abruptly, and it can be used in order to better visualize edges of membrane invaginations. The phase images of erythrocyte ghosts are shown in Figure 4A (parts A5 and A6) for sham and DexP loaded bovine erythrocyte ghosts, respectively, and in Figure 4B (parts B5 and B6) for sham and DexP loaded porcine erythrocyte ghosts, respectively. The phase lag of the AFM cantilever across ghosts' surfaces is represented by a bright contrast. On the other hand, the edges of membrane invaginations can be clearly detected as dark features inside ghosts' surfaces, since these parts correspond to the maximal phase lag of AFM cantilever compared to the ghosts' surfaces.

According to these AFM images, the sham encapsulated bovine erythrocyte ghosts contained considerable amount of surface area with membrane invaginations, having RMS value for the surface roughness of $17.2 \pm 1.4 \mathrm{~nm}$. The encapsulation of DexP led to a slight, but statistically insignificant $(P>0.05)$ decrease in RMS value for the surface roughness which amounted $15.9 \pm 2.8 \mathrm{~nm}$. In the case of DexP loaded porcine erythrocyte ghosts, more prominent defects in erythrocyte ghosts' morphology corresponding to increase in surface roughness were observed. RMS value for the surface roughness amounted $30.0 \pm 5.4 \mathrm{~nm}$ and $34.26 \pm 7.5 \mathrm{~nm}$ for sham and DexP encapsulated porcine ghosts, respectively $(P>0.05)$. 

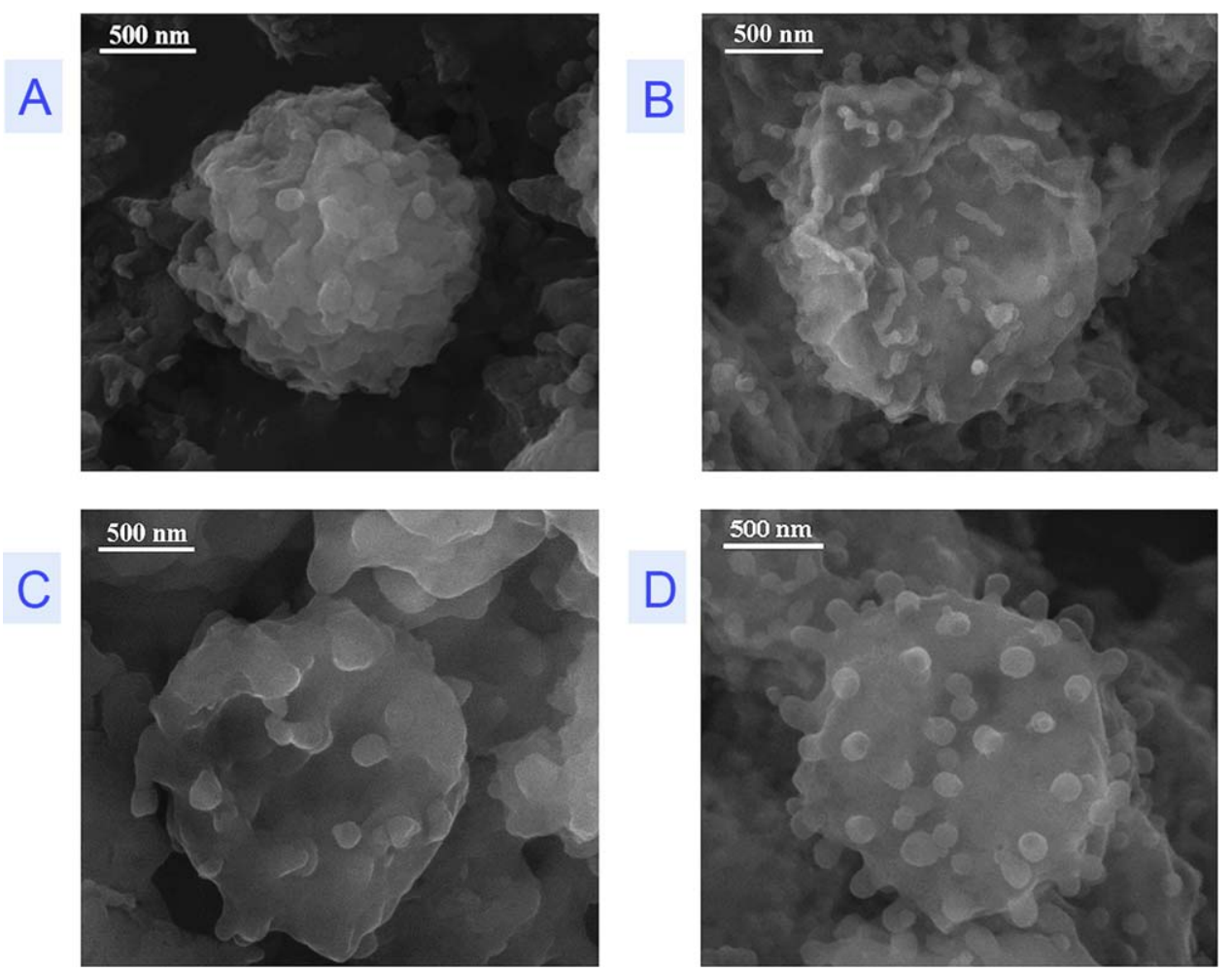

Figure 3. Scanning electron micrographs of (A) sham encapsulated bovine erythrocyte ghosts, (B) DexP encapsulated bovine erythrocyte ghosts, (C) sham encapsulated porcine erythrocyte ghosts, and (D) DexP encapsulated porcine erythrocyte ghosts.
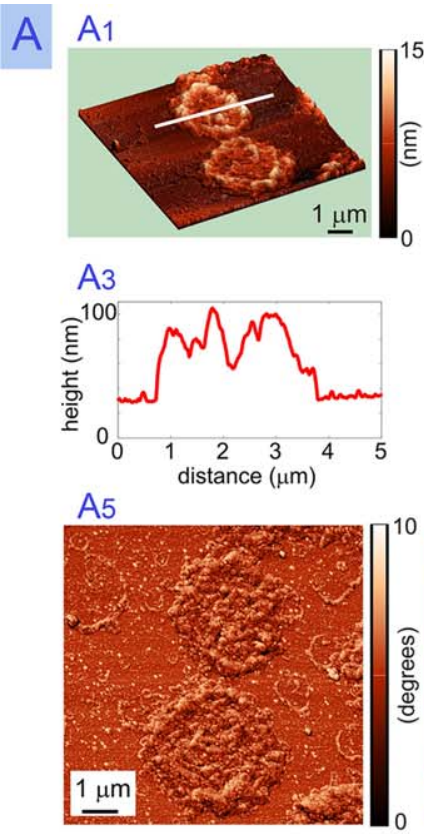
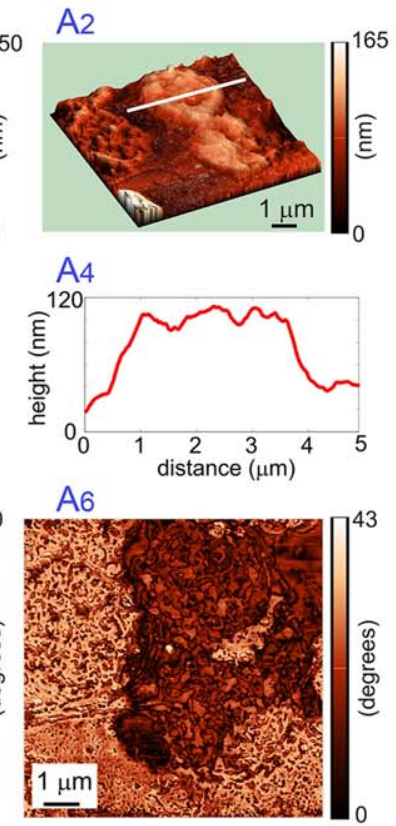
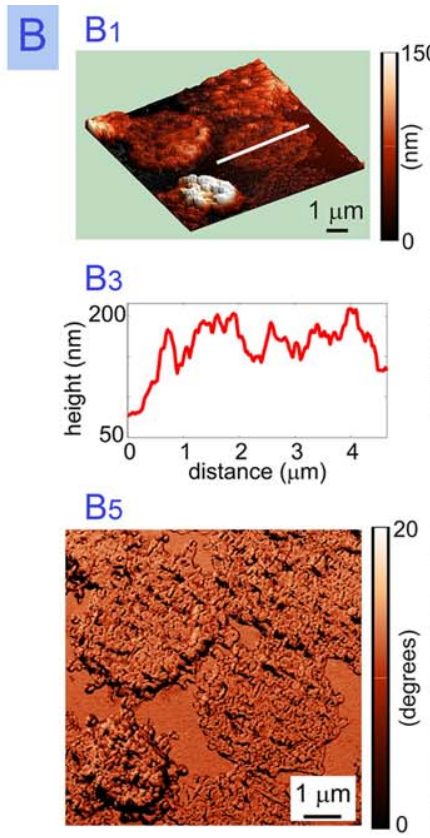

B2
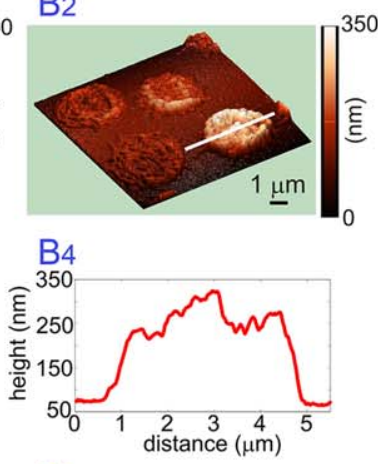

B6

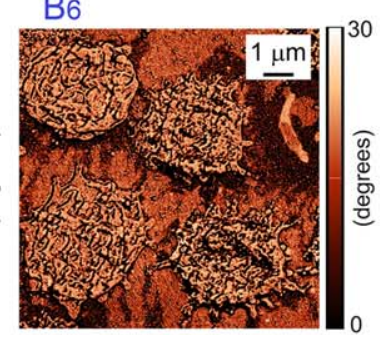

Figure 4. (A) AFM analysis of DexP encapsulation procedure impact on morphology of bovine (A1) Sham encapsulated erythrocyte ghosts, (A2) DexP encapsulated erythrocyte ghosts. (B) AFM analysis of DexP encapsulation procedure impact on morphology of porcine (B1) Sham encapsulated erythrocyte ghosts; (B2) DexP encapsulated erythrocyte ghosts. Parts A1, A2, B1, and $\mathrm{B} 2$ show three-dimensional topographies. Parts A3, A4, B3, and B4 depict the height profile along the lines denoted in parts A1, A2, and B1, B2, respectively. Parts A5, A6, B5, and B6 represent AFM phase images obtained simultaneously with the topographic images.

The AFM topography images along with the calculated RMS value for the surface roughness confirmed described morphological changes for both type of ghosts observed by FE-SEM, indicating that DexP inclusion itself had no observable additional effect on the morphology of the ghosts carriers. However, AFM analysis revealed significant difference in membrane surface roughness between bovine and porcine erythrocyte ghosts (with or without encapsulated 

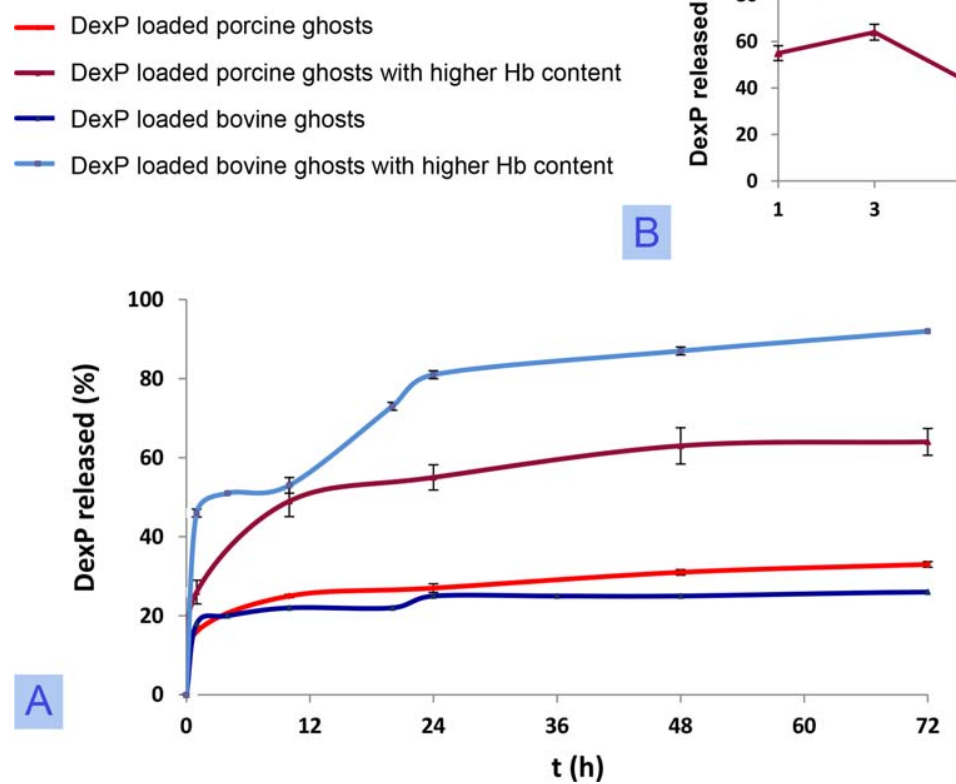

Figure 5. The release profiles of DexP from bovine and porcine erythrocyte ghost carriers. The release profiles in first $72 \mathrm{~h}(\mathrm{~A})$ and from Day 1 up to Day 11 (B). Values are mean \pm SD $(n=3)$.

drug, $P<0.05)$; this could have impact on other distinct properties and behavior of two investigated kind of ghosts as drug carriers.

\section{In vitro drug release}

In vitro drug release assay of DexP-loaded bovine and porcine ghosts was performed under physiological conditions (PBS, pH 7.4), at $37^{\circ} \mathrm{C}$. As already mentioned, the presence of dephosphorylated form of drug, Dex, was not recorded in either bovine or porcine ghosts during release studies by developed UHPLC/DAD/-HESI-MS/MS method. This undoubtedly confirms the absence of conversion of DexP to Dex shown in the case of intact erythrocytes as drug carriers. ${ }^{15}$

In general, kinetics of the drug release from erythrocyte carriers depends on the size and polarity of the encapsulated agents. ${ }^{39}$ Three different patterns of drug release are possible: (i) the drug release occurs by rapid diffusion through the membrane for lipophilic drugs such as primaquin ${ }^{45}$ or methotrexate $^{46}$; (ii) the drug release occurs by cell lysis, i.e., hemoglobin release for polar drugs such as enalaprilat, ${ }^{27}$ gentamicin, ${ }^{47}$ and tramadol $^{37}$; and (iii) the release profile is intermediate between the first two patterns, as observed for erythropoietin ${ }^{48}$ and isoniazid. ${ }^{49}$ Although erythrocyte ghosts represent product of controlled hemolysis of erythrocytes (i.e., the majority of hemoglobin content is removed), some residual hemoglobin is always present in resulting bovine and porcine erythrocyte ghosts. ${ }^{24}$ The level of remained hemoglobin in both bovine and porcine erythrocytes ghosts showed individual variation. In order to investigate its effect on release kinetics, samples of DexP loaded ghosts, containing low residual hemoglobin level (relative amount of $0.0004 \pm 0.0000$ in bovine and $0.0012 \pm 0.0001$ in porcine ghosts), were compared with bovine and porcine samples with higher hemoglobin content $(0.0020 \pm 0.0001$ and $0.0030 \pm 0.0001$, respectively).
The release profiles of all samples are presented in Figure 5. As can be seen, the initial burst associated with surfacebound drug release is more pronounced in case of bovine ghosts release curves. Moreover, the release curves of bovine ghosts exhibit multiphase release pattern. Thus, the initial burst (which lasted four hours) is followed by slow lag phase (which lasted from six to fourteen hours depending on hemoglobin content) and then second burst release phase. The existence of lag-phase indicates that, apart from drug diffusion, some structural changes of the cell membranes were involved, too. When comparing the release patterns of the two types of ghosts, DexP loaded porcine erythrocyte ghosts had lower efflux of drug during the initial burst, and the lack of second burst release phase (Figure 5A). It can also be seen that higher DexP efflux rate was obtained in case of samples with higher hemoglobin content (during the same experimental time) compared to samples with lower remained hemoglobin content and the effect of residual hemoglobin was more pronounced in case of bovine erythrocyte ghosts. This fact supports the theory that drug release is enhanced by hemoglobin release from erythrocyte carriers. $^{27,37}$

Previously we have reported that membrane content and related membrane transport characteristic of bovine and porcine erythrocyte ghosts isolated by gradual hemolysis are closely similar to those of normal erythrocytes. ${ }^{24}$ According to this, the demonstrated differences in DexP release profiles from bovine and porcine ghosts could be related to different efflux transporters in erythrocyte membranes (which mediate drug active efflux out of the membranes), as some authors have been already suggested. ${ }^{36,50}$ Demonstrated nondetection of conversion of DexP to Dex in both types of loaded ghosts could have important contribution to the shown sustained release of dexamethasone, since DexP achieves its therapeutic effect after being transformed to Dex at targeted site.

Another interesting phenomenon was observed in both types of examined ghosts. Namely, after 3-5 days of prolonged release DexP concentration in the medium started to 
decline (Figure 5B). This indicates destabilizing interactions with structural components of erythrocyte membranes under experimental condition used for the release test. Similar observations, declining trend of drug release from erythrocyte carriers have been noticed for other drugs, e.g. interpheron alpha $2 \mathrm{~b}$ and tramadol. ${ }^{36,37}$ Considering other prolonged drug delivery system for DexP, fluctuations in DexP release have been also observed in the case of long circulating liposomes, ${ }^{51}$ but the authors did not rationalize the observed effect.

Regarding the demonstrated features of DexP encapsulated bovine and porcine erythrocyte ghosts it seems that they could have a future in drug delivery. This includes even the field of nano-drug delivery systems, since the erythrocyte ghosts represent starting material in production of nanoerythrosomes as reported recently. ${ }^{52,53}$ However, real biomedical applications of these DexP loaded erythrocyte ghosts will be demonstrated throughout future in vitro and in vivo investigation.

\section{Conclusion}

Developed UHPLC/-HESI-MS/MS based analytical procedure in this study, allowed us to unambiguously identify and quantify DexP encapsulated in bovine and porcine erythrocyte ghosts, to optimize the loading parameters, and to track DexP release kinetics. The EE was approximately fivefold higher in porcine compared to bovine ghosts. Regardless the type of ghosts, incubation at $37^{\circ} \mathrm{C}$ significantly increased loaded amount of DexP. Besides, the loading of DexP into both types of ghosts was directly proportional to drug concentration in the incubation medium. The differences between bovine and porcine DexP loaded ghosts were also observed in drug release kinetics. The sustained drug release from DexP loaded ghosts was shown over 3 days from porcine ghosts and 5 days from bovine ghosts. In the both types of loaded ghosts the conversion of DexP to Dex was not detected. Having strong influence on drug release kinetics, the residual hemoglobin in the ghosts appeared as a new parameter necessary to be controlled in order to have standardized production of bovine and porcine erythrocyte ghosts based systems for prolonged release of DexP. However, real potential of these DexP loaded erythrocyte ghosts as cell-like bio-derived DexP delivery platforms for biomedical applications will be demonstrated throughout future in vitro and in vivo experiments.

\section{Acknowledgment}

The authors are highly acknowledged to Ministry of Education, Science and Technological Development of the Republic of Serbia (Project No. III 46010 and OI 171005, 2011-2015) for financial support. The authors declare that they have no conflict of interest. The authors thank to Danijela Lukić, DVM for having kindly provided us blood samples from the slaughterhouse PKB "Imes" Belgrade, Serbia. Also, authors are very gratefully to Slavko Mojsilović for performing flow-cytometry analysis.

\section{Literature Cited}

1. Rang HP, Dale MM, Ritter JM, Moore PK. The pituitary and adrenal cortex. In: Hardman G, Limbird LE, editors. Pharmacology, 5th ed. New York: Churchill Livingstone, 2003.
2. Ding S, Li J, Luo C, Li, Long L, Yang G, Zhou S. Synergistic effect of released dexamethasone and surface nanoroughness on mesenchymal stem cell differentiation. Biomater Sci. 2013;1: $1091-1100$

3. Gaharwar AK, Mihaila Kulkarni SMAA, Patel A, Di Luca A, Reis RL, Gomes ME, van Blitterswijk C, Moroni L, Khademhosseini A. Amphiphilic beads as depots for sustained drug release integrated into fibrillar scaffolds. J Control Release. 2014;187:66-73.

4. Rossi L, Serafini S, Cenerini L, Picardi F, Bigi L, Panzani I, Magnani M. Erythrocyte-mediated delivery of dexamethasone in patients with chronic obstructive pulmonary disease. Biotechnol Appl Biochem. 2001;33:85-89.

5. Rossi L, Castro M, D’Orio F, Damonte G, Serafini S, Bigi L, Panzani I, Novelli G, Dellapiccola B, Panunzi S, Di Carlo P, Bella S, Magnani M. Low doses of dexamethasone constantly delivered by autologous erythrocytes slow the progression of lung disease in cystic fibrosis patients. Blood Cell Mol Dis. 2004:33:57-63.

6. Eroglu H, Kas HS, Oner L, Turkoglu OF, Akalan N, Sargon MF, Ozer N. The in-vitro and in-vivo characterization of PLGA: L-PLA microspheres containing dexamethasone sodium phosphate. J Microencapsul. 2001;185:603-612.

7. Wang Y, Gao X, Kuriyava S, Bourne D, Grady B, Chen K, Dormer K, Kopke RD. Incorporation, release and effectiveness of dexamethasone in poly(lactic-co-glycolic acid) nanoparticles for inner ear drug delivery. J Nanotechnol Eng Med. 2011;2: 011013-1-011013-7.

8. Yoo J-W, Irvine DJ, Discher DE, Mitragotri S. Bio-inspired, bioengineered and biomimetic drug delivery carriers. Nat Rev Drug Discov. 2011;10:521-535.

9. Annese V, Latiano A, Rossi L, Lombardi G, Dallapiccola B, Serafini S, Damonte G, Andriulli A, Magnani M. Erythrocytesmediated delivery of dexamethasone in steroid-dependent IBD patients - a pilot uncontrolled study. Am J Gastroenterol. 2005; 100:1370-1375.

10. Annese V, Latiano A, Rossi L, Bossa F, Damonte G, Dallapiccola B, Serafini S, Pierige F, Andriulli A, Magnani M. The polymorphism of multi-drug resistance 1 gene (MDR1) does not influence the pharmacokinetics of dexamethasone loaded into autologous erythrocytes of patients with inflammatory bowel disease. Eur Rev Med Pharmacol Sci. 2006;10:2731.

11. Bossa F, Latiano A, Rossi L, Magnani M, Palmieri O, Dallapiccola B, Serafini S, Damonte G, De Santo E, Andriulli A, Annese V. Erythrocyte-mediated delivery of dexamethasone in patients with mild-to-moderate ulcerative colitis, refractory to mesalamine: a randomized, controlled study. Am J Gastroenterol. 2008; 103:2509-2516.

12. Castro M, Rossi L, Papadatou B, Bracci F, Knafelz D, Ambrosini M, Calce A, Serafini S, Isacchi G, D'Orio F. Longterm treatment with autologous red blood cells loaded with dexamethasone 21-phosphate in pediatric patients affected by steroid-dependent Crohn disease. J Pediatr Gastroenterol Nutr. 2007;44:423-426.

13. Bossa F, Annese V, Valvano M, Latiano A, Martino G, Rossi L, Magnani M, Palmieri O, Serafini S, Damonte G, De Santo E, Andriulli A. Erythrocytes-mediated delivery of dexamethasone 21-phosphate in steroid-dependent ulcerative colitis: a randomized, double-blind Sham-controlled study. Inflam Bowel Dis. 2013;19:1872-1879.

14. Chessa L, Leuzzi V, Plebani A, Soresina A, Micheli R, D’Agnano D, Venturi T, Molinaro A, Marini M, Leali PF, Quinti I, Cavaliere FM, Girelli G, Pietrogrande MC, Finocchi A, Tabolli S, Abeni D, Magnani M. Intra-erythrocyte infusion of dexamethasone reduces neurological symptoms in ataxia teleangiectasia patients: results of a phase 2 trial. Orphanet $J$ Rare Dis. 2014;9:5. doi: 10.1186/1750-1172-9-5.

15. Biagiotti S, Paoletti MF, Fraternale A, Rossi L, Magnani M. Drug delivery by red blood cells. IUBMB Life. 2011;63:621631.

16. Lautenschläger C, Schmidt C, Fischer D, Stallmach A. Drug delivery strategies in the therapy of inflammatory bowel disease. Adv Drug Deliv Rev. 2014;71:58-76. 
17. Mansouri S, Merhi Y, Winnik FM, Tabrizian M. Investigation of layer-by-layer assembly of polyelectrolytes on fully functional human red blood cells in suspension for attenuated immune response. Biomacromolecules. 2011;12:585-592.

18. Cerda-Cristerna BI, Cottin S, Flebus L, Pozos-Guillén A, Flores $\mathrm{H}$, Heinen E, Jolois O, Gérard C, Maggipinto G, Sevrin C, Grandfils C. Poly(2-dimethylamino ethylmethacrylate)-based polymers to Camouflage red blood cell antigens. Biomacromolecules. 2012;13:1172-1180.

19. Wei J, Chiang B. Bioactive peptide production by hydrolysis of porcine blood proteins in a continuous enzymatic membrane reactor. J Sci Food Agric. 2009;89:372-378.

20. Hu J, Xu M, Hang B, Wang L, Wang Q, Chen J, Song T, Fu D, Wang Z, Wang S, Sanhu L, Liu X. Isolation and characterization of an antimicrobial peptide from bovine hemoglobin $\alpha$-subunit. World J Microb Biot. 2011;27:767-771.

21. Stojanović R, Ilić V, Manojlović V, Bugarski D, Dević M, Bugarski B. Isolation of hemoglobin from bovine erythrocytes by controlled hemolysis in the membrane bioreactor. Appl Biochem Biotechnol. 2012;166:1491-1506.

22. DeLoach JR, Harris RL, Ihler GM. An erythrocyte encapsulator dialyzer used in preparing large quantities of erythrocyte ghosts and encapsulation of a pesticide in erythrocyte ghosts. Anal Biochem. 1980;102:220-227.

23. DeLoach JR, Spates GE. A comparison of membrane lipid content of normal and carrier-erythrocytes from cattle. Ital J Biochem. 1988;37:386-391.

24. Kostić IT, Ilić VLj, Đorđević VB, Bukara KM, Mojsilović SB, Nedović VA, Bugarski DS, Veljović ĐN, Mišić DM, Bugarski BM. Erythrocyte membranes from slaughterhouse blood as potential drug vehicles: isolation by gradual hypotonic hemolysis and biochemical and morphological characterization. Colloids Surf B, Biointerfaces. 2014;122:250-259.

25. Martín-Sabroso C, Tavares-Fernandes DF, Espada-García JI, Torres-Suárez AI. Validation protocol of analytical procedures for quantification of drugs in polymeric systems for parenteral administration: dexamethasone phosphate disodium microparticles. Int J Pharm. 2013;458:188-196.

26. Rivas IP, Gil-Alegre ME, Torres-Suarez AI. Development and validation of a fast high-performance liquid chromatography method for the determination of microencapsulated pyrethroid pesticides. Anal Chim Acta. 2006;557:245-251.

27. Hamidi M, Tajerzadeh H, Dehpour AR, Rouini MR, Ejtemaee MS. In vitro characterization of human intact erythrocytes loaded by enalaprilat. Drug Deliv. 2001;8:223-230.

28. Liu H, Chen X, Zhang S, Qu L, Zhao Y, Liu H, Dong M. Separation and determination of dexamethasone sodium phosphate in cochlear perilymph fluid by liquid chromatography with ultraviolet monitoring and electrospray ionization mass spectrometry characterization, J Chrom B. 2004;805:255-260.

29. Nelson GJ. Composition of neutral lipids from erythrocytes of common mammals. J Lipid Res. 1967;8:374-379.

30. Virtanen JA, Cheng KH, Somerharju P. Phospholipid composition of the mammalian red cell membrane can be rationalized by a superlattice model. Proc Natl Acad Sci U S A. 1998;95: 4964-4969.

31. Ferlazzo AM, Bruschetta G, Di Pietro P, Medica P, Notti A, Rotondo E. Phospholipid composition of plasma and erythrocyte membranes in animal species by ${ }^{31} \mathrm{P}$ NMR. Vet Res Commun. 2011;35:521-530.

32. Florin-Christensen J, Suarez CE, Florin-Christensen M, Wainszelbaum M, Brown WC, McElwain TF, Palmer GH. A unique phospholipid organization in bovine erythrocyte membranes. Proc Natl Acad Sci USA. 2001;98:7736-7741.

33. Webb MS, Harasym TO, Masin D, Bally MB, Mayer LD. Sphingomyelin-cholesterol liposomes significantly enhance the pharmacokinetic and therapeutic properties of vincristine in murine and human tumour models. Br J Cancer. 1995;72:896904.

34. Millan GC, Bax BE, Castaneda AZ, Marinero ML, Lanao JM. In vitro studies of amikacin-loaded human carrier erythrocytes. Transl Res. 2008;152:59-66.
35. Hamidi M, Zarei N, Zarrin AH, Mohammadi-Samani S. Preparation and in vitro characterization of carrier erythrocytes for vaccine delivery. Int J Pharm. 2007;338:70-78.

36. Hamidi M, Zarrin AH, Foroozesh M, Zarei N, MohammadiSamani S. Preparation and in vitro evaluation of carrier erythrocytes for RES-targeted delivery of interferon-alpha $2 \mathrm{~b}$. Int $J$ Pharm. 2007;341:125-133.

37. Foroozesh M, Hamidi M, Zarrin A, Mohammadi-Samani S, Montaseri H. Preparation and in-vitro characterization of tramadol-loaded carrier erythrocytes for long-term intravenous delivery, J Pharm Pharmacol. 2011;63:322-332.

38. Millán CG, Marinero MLS, Castañeda AZ, Lanao JM. Drug, enzyme and peptide delivery using erythrocytes as carriers. J Control Release. 2004;95:27-49.

39. Shaillender M, Luo R, Venkatraman SS, Neu B. Layer-by-layer microcapsules templated on erythrocyte ghost carriers. Int $J$ Pharm. 2011;415:211-217.

40. Staedtke V, Brähler M, Müller A, Georgieva R, Bauer S, Sternberg N, Voigt A, Lemke A, Keck C, Möschwitzer J, Bäumler $H$. In vitro inhibition of fungal activity by macrophage-mediated sequestration and release of encapsulated Amphotericin B nanosupension in red blood cells. Small. 2010; 6:96-103.

41. Kim SH, Kim EJ, Hou JH, Kim JM, Choi HG, Shim CK, Oh YK. Opsonized erythrocyte ghosts for liver-targeted delivery of antisense oligodeoxynucleotides. Biomaterials. 2009;30:959967.

42. Sternberg N, Georgieva R, Duft K, Bäumler H. Surface-modified loaded human red blood cells for targeting and delivery of drugs. J Microencapsul. 2012;29:9-20.

43. Jaraswekin S, Prakongpan S, Bodmeier R. Effect of poly(lactide-co-glycolide) molecular weight on the release of dexamethasone sodium phosphate from microparticles. J Microencapsul. 2007;242:117-128.

44. Girasole M, Pompeo G, Cricenti A, Longo G, Boumis G, Bellelli A, Amiconi S. The how, when, and why of the aging signals appearing on the human erythrocyte membrane: an atomic force microscopy study of surface roughness. Nanomedicine. 2010;6:760-768.

45. Talwar N, Jain NK. Erythrocyte based delivery system of primaquine: in vitro characterization. J Microencapsul. 1992;9:357364.

46. Lewis DA, Alpar HO. Therapeutic possibilities of drugs encapsulated in erythrocytes, Int J Pharm. 1984;22:137-146.

47. Eichler HG, Gasic S, Bauer K. In vivo clearance of antibodysensitized human drug carrier erythrocytes. Clin Pharmacol Ther. 1986;40:300-303.

48. Garín MI, López RM, Sanz S, Pinilla M, Luque J. Erythrocytes as carriers for recombinant human erythropoietin. Pharm Res. 1996; 13:869-874.

49. Jain S, Jain SK, Dixit VK. Erythrocytes based delivery of isoniazid: preparation and in-vitro characterization. Indian Drugs. 1995;32:471-476.

50. Harisa G-D, Ibrahim MF, Alanazi FK. Characterization of human erythrocytes as potential carrier for pravastatin: an in vitro study. Int J Med Sci. 2011;8:222-230.

51. Quan L, Zhang Y, Crielaard BJ, Dusad A, Lele SM, Rijcken CJ, Metselaar JM, Kostková H, Etrych T, Ulbrich K, Kiessling F, Mikuls TR, Hennink WE, Storm G, Lammers T, Wang D. Nanomedicines for inflammatory arthritis: head-to-head comparison of glucocorticoid-containing polymers, micelles, and liposomes. ACS Nano. 2014;8:458-466.

52. Gupta N, Patel B, Ahsan F. Nano-engineered erythrocyte ghosts as inhalational carriers for delivery of fasudil: preparation and characterization. Pharm Res. 2014;31:1553-1565.

53. Harisa GI, Badran MM, Alanazi FK, Erythrocyte nanovesicles: biogenesis, biological roles and therapeutic approach: Erythrocyte nanovesicles. Saudi Pharm J. 2015, http://dx.doi.org/10. 1016/j.jsps.2015.06.010

Manuscript received Nov. 1, 2015, and revision received Feb. 2, 2016. 